\title{
Avaliação de um programa para computador de mão no auxílio ao ensino de oftalmologia para estudantes de medicina
}

\author{
Evaluation of a ophthalmology learning support \\ for handheld computer for medical students
}

Vladimir Camelo Pinto ${ }^{1}$, Thiago Martini da Costa ${ }^{2}$, Gustavo Teixeira Grottone ${ }^{3}$, Paulo Schor ${ }^{4}$, Ivan Torres Pisa ${ }^{5}$

\section{Resumo}

Objetivo:Na última década os computadores de mão se popularizaram entre profissionais da saúde. Nos EUA, por exemplo, cerca de 60-70\% dos estudantes de medicina já os utilizam. A disponibilização de informação clínica nestes dispositivos pode auxiliar no ensino de práticas médicas, no entanto, a maneira como são estruturadas pode influenciar na satisfação e no aprendizado dos alunos. Portanto, este artigo objetiva apresentar os resultados da avaliação do MDFluxo: um programa para auxílio ao ensino de oftalmologia em computadores de mão. Métodos: $\mathrm{O}$ programa foi desenvolvido com fluxogramas estruturados, extraídos do Guia de Oftalmologia da Universidade Federal de São Paulo. A avaliação foi realizada, comparando o desempenho de 20 estudantes de medicina na solução de casos clínicos, utilizando o programa, o livro ou nenhum material. Resultados: Os resultados mostram que o desempenho dos estudantes na avaliação com o livro foi equivalente ao MDFluxo nas questões sobre etiologia $(p<0,01)$ e conduta $(\mathrm{p}<0,05)$ na solução dos casos clínicos, desempenho melhor do que sem a utilização de nenhum material. Conclusão: Na avaliação de satisfação com o uso do programa $82,5 \%$ dos estudantes aprovou sua utilização na solução de casos clínicos.

Descritores: Computadores de mão; Estudantes de Medicina; Oftalmologia; Avaliação de desempenho

\footnotetext{
${ }^{1}$ Mestre, Colaborador do Departamento de Informática em Saúde (DIS) da Universidade Federal de São Paulo - UNIFESP - São Paulo (SP), Brasil;

²ós-graduando (Mestrado) em Informática em Saúde, Departamento de Informática em Saúde (DIS) da Universidade Federal de São Paulo - UNIFESP - São Paulo (SP), Brasil;

${ }^{3}$ Pós-graduando (Doutorado) em Oftalmologia, Departamento de Oftalmologia da Universidade Federal de São Paulo - UNIFESP São Paulo (SP), Brasil;

${ }^{4}$ Livre-docente, Professor Adjunto do Departamento de Oftalmologia da Universidade Federal de São Paulo - UNIFESP - São Paulo (SP), Brasil;

${ }^{5}$ Doutor, Professor Adjunto do Departamento de Informática em Saúde (DIS) da Universidade Federal de São Paulo - UNIFESP - São Paulo (SP), Brasil.
}

Trabalho realizado no Departamento de Informática em Saúde (DIS) da Universidade Federal de São Paulo - UNIFESP - São Paulo (SP), Brasil.

Conflito de Interesses

Este projeto de pesquisa de mestrado foi submetido ao Comitê de Ética em Pesquisa (CEP) da Universidade Federal de São Paulo (UNIFESP), obtendo parecer favorável à sua realização, em 17 de março de 2006 sob o código CEP 0236/06.

Os autores afirmam não haver qualquer conflito de interesse nesta pesquisa. Este projeto não foi aplicado em pacientes, não apresentando fatores de riscos e está isento de quaisquer fins econômicos. 


\section{INTRODUÇÃO}

$\mathbf{0}$ $\mathrm{s}$ assistentes digitais pessoais (personal digital assistants - PDAs), também conhecidos como computadores de mão, se popularizaram na última década entre os profissionais da área da saúde e estudantes de medicina ${ }^{(1,2)}$ por apresentar boa capacidade de armazenamento, acesso rápido a informações, possibilitando maior flexibilidade em ambiente hospitalar se comparado ao uso do computador pessoal (PC) $)^{(3,4)}$.

Segundo Grasso et al. ${ }^{(5)}$, aproximadamente $60 \%$ a $70 \%$ dos estudantes de medicina e médicos residentes nos Estados Unidos utilizavam computadores de mão para propósitos educacionais, sendo considerados como um valioso recurso educacional no ensino de práticas médicas $^{(6)}$ como, por exemplo, na Indiana University School of Medicine (IUSM), onde o uso do computador de mão por estudantes do $3^{\circ}$ ano de medicina já é obrigatório durante suas visitas ambulatórias para registrar suas experiências clínicas ${ }^{(7)}$.

Para observar o uso dos computadores de mão na área da saúde, como ferramenta de auxílio ao ensino, foi realizada uma revisão na literatura médica na base de dados do PubMed $\AA$ - National Center for Biotechnology Information (NCBI) (http://www.ncbi.nlm.nih.gov). Para esta pesquisa foram selecionados um conjunto de descritores de ciência da saúde pesquisados no Medical Subject Headings (MeSH), disponíveis na mesma base de dados.

Nesta revisão foram selecionados um total de 4.775 resumos de artigos com data de publicação entre março de 2004 a dezembro de 2006, com estudos sobre a aplicação dos computadores de mão na área da saúde e no auxílio ao ensino entre estudantes de medicina. Para a inclusão dos artigos completos foram aplicados critérios de inclusão e exclusão nos resumos selecionados, sendo aceitos na revisão 59 artigos completos.

Por meio desta revisão foi observado que o uso dos computadores de mão pode apresentar potencial para:

Disponibilizar informações clínicas com praticidade em ambiente hospitalar ${ }^{(4)}$;

Auxiliar no aprendizado e memorização de rotinas e procedimentos clínicos em ambiente hospitalar ${ }^{(8,9)}$;

Auxiliar a prática médica em diferentes especialidades clínicas ${ }^{(2,10-12)}$;

Ofertar dados e informações clínicas do paciente onde o acesso é restrito ou difícil ${ }^{(13)}$; clínicas $^{(14)}$;
Minimizar possíveis erros na entrada de dados se comparado ao uso do método tradicional baseado em papel e caneta ${ }^{(14)}$.

Nesta revisão percebeu-se que os computadores de mão são utilizados por profissionais da saúde principalmente como ferramentas de pesquisa a informações clínicas $^{(15)}$ e para agendar consultas com pacientes ${ }^{(8)}$. Os programas mais citados na literatura médica e utilizados por profissionais da área da saúde e estudantes de medicina são: calculadoras médicas ${ }^{(8,16-18)}$, referências bibliográficas ${ }^{(19,20)}$, banco de dados de medicamen$\operatorname{tos}^{(18.21,22)}$, programas para observação de imagens médicas $^{(23,25)}$ e outros.

Em um estudo realizado por Grad et al. ${ }^{(26)}$, o uso de informações clínicas somente em formato texto, sem estruturação, em um computador de mão pode ter influenciado na compreensão do conteúdo pelos estudantes de medicina.

Para utilizar a informação clínica por meio de um computador de mão deve ser considerada a forma como esta será apresentada ao usuário e sua estruturação. $\mathrm{Na}$ literatura a informação clínica é apresentada como uma descrição de uma lesão. No livro Guia de Oftalmologia da Universidade Federal de São Paulo - Escola Paulista de Medicina (UNIFESP-EPM) de Schor et al.(27) é apresentado um conjunto de informações clínicas estruturadas. Cada capítulo apresenta uma descrição das lesões mais comuns em cada segmento do olho humano e sua localização anatômica. Ao final de cada capítulo, um fluxograma resume o conhecimento médico discutido.

Esta forma de representação da informação clínica por meio de fluxogramas apresenta um processo mnemônico de maneira a auxiliar o leitor a fixar o conteúdo do texto de forma prática e objetiva. Porém, os fluxogramas do livro ${ }^{(27)}$ não apresentam um mesmo padrão lógico único de formatação entre si, pois cada capítulo do livro foi escrito por um colaborador diferente e o fluxograma vinculado ao capítulo foi construído sem o uso de um modelo de referência, baseando-se apenas na experiência profissional do responsável por sua especificação.

Disponibilizar estes fluxogramas do livro $^{(27)} \mathrm{em}$ formato digital por meio de computadores de mão possibilita uma utilização rápida e de forma interativa destas informações, auxiliando a educação médica entre estudantes de medicina ${ }^{(26)}$. Segundo Kara-José(28) os conhecimentos básicos de Oftalmologia são necessários para o dia a dia de diversas especialidades médicas devido ao frequente envolvimento ocular em doenças sistêmicas. Para disponibilizar estes fluxogramas em formato digital ${ }^{(26)}$, deve ser especificado um modelo único 


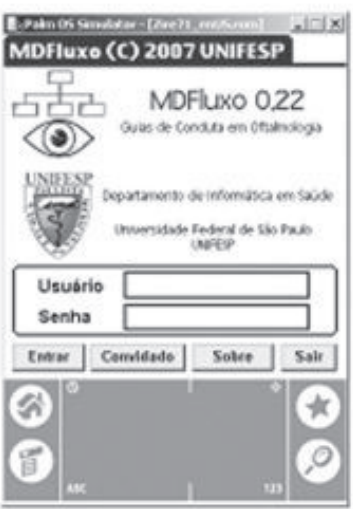

(a)

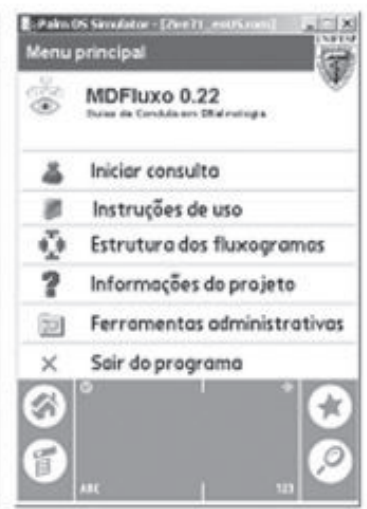

(b)

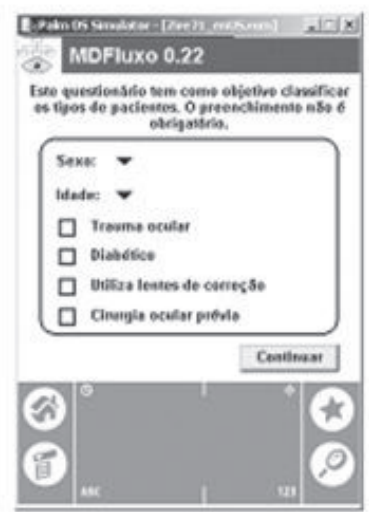

(c)

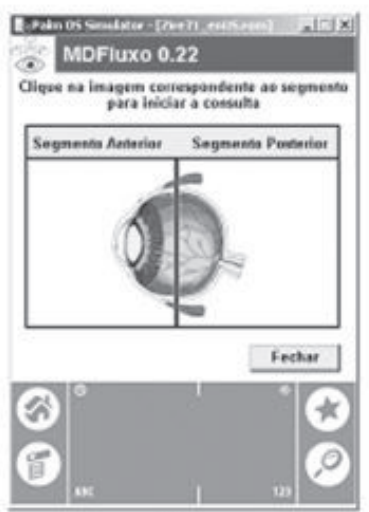

(d)

Figura 1: Telas do programa MDFluxo versão 0.22 utilizada na avaliação de desempenho: (a) login; (b) menu principal; (c) questionário inicial; e (d) categoria de fluxogramas

para representar as informações com uma mesma estrutura facilitando sua digitalização.

O objetivo deste artigo é apresentar os resultados da avaliação do programa aqui denominado Modelo Digital de Fluxogramas em Oftalmologia (MDFluxo), para computadores de mão Palm ${ }^{\circledR}$ com os fluxogramas do livro ${ }^{(27)}$ em formato digital focado na solução de casos clínicos para auxiliar o ensino em oftalmologia para estudantes de medicina.

\section{Métodos}

A construção do modelo para representar os fluxogramas do livro ${ }^{(27)}$ com uma mesma estruturação foi realizada em conjunto com especialistas do departamento de oftalmologia da UNIFESP. Os fluxogramas representados com uma mesma estrutura foram digitalizados utilizando o formato eXtensible Markup Language $(\mathrm{XML})^{(26)}$, para possibilitar seu uso em diferentes ambientes computacionais. $\mathrm{O}$ formato XML utilizado baseiase no Guideline Element Model (GEM) ${ }^{(30)}$, que melhor representa a heterogeneidade contida nas diretrizes que representam a prática médica ${ }^{(30)}$.

Para a construção do programa foi utilizado o plugin MobileVB 4.0 da AppForge ${ }^{\circledR}$ integrado ao Microsoft Visual Basic ${ }^{\circledR}$ 6.0.A metodologia utilizada no desenvolvimento do programa foi a eXtreme Programming (XP) ${ }^{(31)}$. Os arquivos Portable Database (PDB), banco de dados aceito para computadores de mão, foram criados a partir de tabelas do Microsoft Access ${ }^{\circledR}$ 2003.

Para avaliar o desempenho do programa foram aplicadas 3 avaliações com 20 estudantes voluntários do $4^{\circ}$ e $5^{\circ}$ ano de medicina da UNIFESP e da Faculdade de Ciências Médicas de Santos - Centro Universitário Lusíadas (FCMS-UNILUS) no período de abril a maio de 2007. Não foi observado se os estudantes de medicina possuíam conhecimento prévio no uso dos computadores de mão, porque o programa MDFluxo tem por objetivo ser intuitivo para o uso pelos estudantes, independente do conhecimento no uso do dispositivo.

Foram apresentados aos estudantes 3 casos clínicos diferentes, porém, equivalentes entre si, um para cada avaliação, elaborados pelo professor de oftalmologia com experiência didática comprovada na instituição, totalizando 60 avaliações.

Cada estudante de medicina realizou uma avaliação alternando a utilização do material de apoio, sendo: uma avaliação utilizando o computador de mão com o programa MDFluxo, uma avaliação utilizando o livro $^{(27)}$ e uma última avaliação sem utilizar qualquer material de apoio. A alternância do material de apoio foi para que todos os estudantes de medicina que participaram do experimento utilizassem o computador de mão com o programa.

Os casos clínicos foram formados pelos seguintes itens: identificação do paciente, a condição clínica apresentada, uma história clínica e os fatores associados. Nestas avaliações os estudantes responderam as seguintes questões na solução do caso clínico: hipótese diagnóstica, etiologia e conduta. Foram atribuídos valores $1 ; 0,5$ e 0 para respostas certas, meio certas e erradas, respectivamente. $\mathrm{O}$ tempo decorrido na avaliação foi anotado para uma análise estatística.

Os estudantes que realizaram a avaliação utilizando como material de apoio o computador de mão com o programa responderam um questionário de ava- 
liação de satisfação sobre o uso do programa durante a avaliação.

O questionário é composto por questões sobre eficácia, eficiência, confiabilidade, consistência, funcionalidade, facilidade, navegabilidade, agilidade, objetividade e qualidade do programa para auxiliar na solução do caso clínico apresentado aos estudantes de medicina, para cada questão os estudantes atribuíram notas de satisfação com o uso do programa nos itens apresentados. As notas são: (1) péssimo, (2) ruim, (3) satisfatório, (4) bom e (5) ótimo.

Para a análise dos dados obtidos na avaliação de desempenho do programa na solução dos casos clínicos em oftalmologia foi aplicado o teste não paramétrico de Friedman ${ }^{(32)}$ para analisar a diferença entre as avaliações, utilizando materiais de apoio diferentes.

\section{Resultados}

Com o auxílio de especialistas do departamento de oftalmologia da UNIFESP foi construído o modelo para representar os fluxogramas do livro(27) e a partir deste, 15 dos 55 fluxogramas foram reestruturados, com base na estrutura especificada no modelo proposto, e destes, 3 tiveram seu conteúdo revisado por especialistas. Estes fluxogramas foram representados em formato XML.

O MobileVB integrado ao Microsoft Visual Basic 6.0 e a utilização da metodologia XP permitiu maior agilidade na construção do programa MDFluxo e uma interação constante com os especialistas sendo criados vários protótipos do programa. A cada protótipo construído validações foram realizadas por especialistas para avaliar e criticar a funcionalidade, interação e layout do programa. Foram construídos 22 protótipos, sendo a versão 0.22 considerada estável para aplicação da avaliação com os estudantes de medicina segundo a opinião do especialista consultado. A Figura 1 apresenta o programa MDFluxo 0.22.

Com a construção do programa MDFluxo, os fluxogramas representados em formato XML foram armazenados em sua base de dados. A base de dados do programa MDfluxo possui um arquivo de log para armazenar cada item selecionado pelo estudante de medicina durante a utilização do programa, gerando um log de ações.

Após a construção e validação do programa MDFluxo e a inserção dos fluxogramas em formato XML na base de dados, foram realizadas as avaliações de desempenho do programa com os estudantes de medicina

\section{da UNIFESP e FCMS-UNILUS.}

Os casos clínicos respondidos pelos estudantes de medicina foram analisados pelo mesmo professor e os dados tabulados em uma planilha eletrônica, sendo estes divididos em hipótese diagnóstica, etiologia, conduta, somatória dos resultados (hipótese diagnóstica + etiologia + conduta) e tempo para finalizar o caso clínico durante a avaliação, como apresentado na Tabela 1.

Nos dados obtidos na avaliação com os estudantes foi aplicado o teste estatístico de Friedman ${ }^{(32)}$ e fixados em 0,05 (5\%) o nível de rejeição da hipótese de nulidade, para observar a diferença entre as avaliações realizadas com a utilização de cada um dos materiais de apoio como apresentado na Tabela 2.

Com base nos resultados do teste estatístico aplicado nos dados da avaliação como apresentado na Tabela 2 é possível observar que:

- Hipótese diagnóstica: não apresentou diferença significativa entre as avaliações realizadas com os estudantes de medicina utilizando o computador de mão com o programa MDFluxo, o livro ${ }^{(27)}$ e sem qualquer material de apoio.

- Etiologia: foi observado entre as avaliações que utilizando o computador de mão, com o programa MDFluxo e o livro ${ }^{(27)}$ como material de apoio, os estudantes obtiveram melhores resultados se comparado a não utilização de nenhum material de apoio, porém o uso do computador de mão com o programa MDFluxo e do livro ${ }^{(27)}$ como material de apoio foram equivalentes entre si.

- Conduta: a utilização do livro ${ }^{(27)}$ como material de apoio apresentou diferença significativa em relação à utilização do computador de mão com o programa MDFluxo e a não utilização de nenhum material de apoio.

- Somatória dos resultados: não apresentou diferença significativa entre as avaliações realizadas com os estudantes de medicina utilizando o computador de mão com o programa MDFluxo, o livro ${ }^{(27)}$ e sem qualquer material de apoio.

- Tempo para finalizar o caso clínico: a utilização do livro ${ }^{(27)}$ apresentou diferença significativa em relação à utilização do computador de mão com o programa MDFluxo e a não utilização de nenhum material de apoio.

A Tabela 3 apresenta os dados do questionário de satisfação com o uso do programa MDFluxo na solução dos casos clínicos com a média das notas atribuídas (valores entre 1 e 5) pelos estudantes durante a utilização do programa na solução dos casos clínicos e suas respectivas porcentagens. 
Tabela 1

Dados obtidos na avaliação de desempenho do programa MDFluxo com os estudantes

\begin{tabular}{|c|c|c|c|c|c|c|c|c|c|c|c|c|c|c|}
\hline \multicolumn{3}{|c|}{$\begin{array}{c}\text { Hipótese } \\
\text { Diagnóstica }\end{array}$} & \multicolumn{3}{|c|}{ Etiologia } & \multicolumn{3}{|c|}{ Conduta } & \multicolumn{3}{|c|}{$\begin{array}{c}\text { Somatória } \\
\text { dos resultados }\end{array}$} & \multicolumn{3}{|c|}{$\begin{array}{c}\text { Tempo para } \\
\text { finalizar o caso clínico }\end{array}$} \\
\hline $\mathbf{C M}$ & Livro & $\mathbf{S} / \mathbf{M}$ & $\mathbf{C M}$ & Livro & $\mathbf{S} / \mathbf{M}$ & $\mathbf{C M}$ & Livro & $\mathbf{S} / \mathbf{M}$ & $\mathbf{C M}$ & Livro & $\mathbf{S} / \mathbf{M}$ & $\mathbf{C M}$ & Livro & $\mathbf{S} / \mathbf{M}$ \\
\hline 1,00 & 1,00 & 0 & 0 & & & 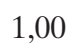 & 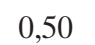 & & 3,00 & 2,50 & 0,50 & $12^{\prime} 50^{\prime \prime}$ & $16^{\prime} 33^{\prime \prime}$ & $7^{\prime} 00^{\prime \prime}$ \\
\hline 1,00 & 0,00 & 1,00 & 0,50 & 0,00 & 0,00 & 1,00 & 0,00 & 0,50 & 2,50 & 0,00 & 1,50 & $11^{\prime} 50^{\prime \prime}$ & $20^{\prime} 00^{\prime \prime}$ & $18^{\prime} 00^{\prime \prime}$ \\
\hline 0,50 & 0,00 & 0,00 & 50 & 0 & 0,00 & 0,00 & & ) & 1,00 & 0,50 & 0,00 & $10^{\prime} 15^{\prime \prime}$ & $32^{\prime}$ & $12^{\prime} 82^{\prime \prime}$ \\
\hline 1,00 & 1,00 & 0,00 & & 0,00 & 0,00 & 1,00 & 0,00 & & 3,00 & 1,00 & 0,00 & $12^{\prime} 00^{\prime \prime}$ & $11^{\prime} 00^{\prime \prime}$ & $8^{\prime} 33^{\prime \prime}$ \\
\hline 0,00 & 1,00 & 1,00 & 0,00 & 0,00 & 0,00 & 0,00 & 0,00 & 0,50 & 0,00 & 1,00 & 1,50 & $16^{\prime} 00^{\prime \prime}$ & $11^{\prime} 00^{\prime \prime}$ & $12^{\prime} 83^{\prime \prime}$ \\
\hline 1,00 & 1,00 & 1,00 & 1,00 & 0,00 & 0,00 & 1,00 & 0,00 & 0,00 & 3,00 & 1,00 & 1,00 & $20^{\prime} 00^{\prime \prime}$ & $24^{\prime} 00^{\prime \prime}$ & $17^{\prime} 33^{\prime \prime}$ \\
\hline 0,50 & & 1,00 & & & 0, & 0 , & & & 0,50 & 1,00 & 1,00 & $16^{\prime} 63^{\prime \prime}$ & $10^{\prime}$ & $19^{\prime} 00^{\prime \prime}$ \\
\hline 0,00 & 0,00 & 0,00 & 0,50 & & 0,00 & 0,00 & 0,00 & 0,00 & 0,50 & 0,50 & 0,00 & $15^{\prime} 02^{\prime \prime}$ & $12^{\prime} 58^{\prime \prime}$ & $3^{\prime} 50^{\prime \prime}$ \\
\hline 0,00 & 0,50 & 0,50 & 0,00 & 0,00 & 0,00 & 0,00 & 0,00 & 0,50 & 0,00 & 0,50 & 1,00 & $14^{\prime} 20^{\prime \prime}$ & $8^{\prime} 50^{\prime \prime}$ & $15^{\prime} 90^{\prime \prime}$ \\
\hline 0,00 & 1,00 & 0,00 & 0,00 & & 0,0 & 0,00 & & & 0,00 & 2,00 & 0,00 & $16^{\prime} 00^{\prime \prime}$ & $9^{\prime} 75^{\prime \prime}$ & $11^{\prime} 20^{\prime \prime}$ \\
\hline 1,00 & & & & & & & & & & & & & & \\
\hline 0,00 & 0,50 & 0,00 & 0,00 & 0,00 & 0,00 & 0,50 & 0,50 & 0,00 & 0,50 & 1,00 & 0,00 & $23^{\prime} 87^{\prime \prime}$ & $16^{\prime} 75^{\prime \prime}$ & $11^{\prime} 58^{\prime \prime}$ \\
\hline 1,00 & 0,50 & 0,00 & 0,50 & 0,50 & 0,00 & 1,00 & 1,00 & 0,00 & 2,50 & 2,00 & 0,00 & $14^{\prime} 00^{\prime \prime}$ & $17^{\prime} 52^{\prime \prime}$ & $10^{\prime} 58^{\prime \prime}$ \\
\hline 1,00 & 1,00 & 1,00 & 1,00 & & 0,00 & 1,00 & 1,00 & 0,00 & 3,00 & 2,00 & 1,00 & $22^{\prime} 00^{\prime \prime}$ & $10^{\prime} 00^{\prime \prime}$ & $12^{\prime} 83^{\prime \prime}$ \\
\hline 0,00 & 0,50 & 0,00 & 0,00 & 0,50 & 0,00 & 0,00 & 0,50 & 0,00 & 0,00 & 1,50 & 0,00 & $12^{\prime} 42^{\prime \prime}$ & $16^{\prime} 00^{\prime \prime}$ & $10^{\prime} 28^{\prime \prime}$ \\
\hline 0,00 & 1,00 & 0,50 & 0,00 & 0,00 & 0,00 & 0,00 & 0,50 & 0,00 & 0,00 & 1,50 & 0,50 & $10^{\prime} 50^{\prime \prime}$ & $7^{\prime} 00^{\prime \prime}$ & 9' $95^{\prime \prime}$ \\
\hline 1,00 & 1,00 & 0,00 & 0,00 & 100 & 0,00 & 0,50 & 1,00 & 0,00 & 1,50 & 3,00 & 0,00 & $23^{\prime} 00^{\prime \prime}$ & $23^{\prime} 75^{\prime \prime}$ & $19^{\prime} 00^{\prime \prime}$ \\
\hline 1,00 & 0,50 & 1,00 & 0,00 & 0,00 & 0,00 & 0,00 & 0,00 & 0,00 & 1,00 & 0,50 & 1,00 & $32^{\prime} 00^{\prime \prime}$ & $13^{\prime} 50^{\prime \prime}$ & $14^{\prime} 42^{\prime \prime}$ \\
\hline 1,00 & 1,00 & 1,00 & 1,00 & 1,00 & 0,00 & 1,00 & 1,00 & 0,00 & 3,00 & 3,00 & 1,00 & $13^{\prime} 25^{\prime \prime}$ & $5^{\prime} 00^{\prime \prime}$ & $10^{\prime} 50^{\prime \prime}$ \\
\hline 1,00 & 0,00 & 1,00 & 0,00 & 0,50 & 0,50 & 0,50 & 0,00 & 0,50 & 1,50 & 0,50 & 2,00 & $21^{\prime} 00^{\prime \prime}$ & $10^{\prime} 50^{\prime \prime}$ & $12^{\prime} 52^{\prime \prime}$ \\
\hline
\end{tabular}

$\mathrm{CM}=$ computador de mão; $\mathrm{S} / \mathrm{M}=$ sem qualquer material de apoio

Tabela 2

Resultado do teste estatístico de Friedman (Siegel \& Castellan, 1988) aplicado nos dados obtidos na avaliação de desempenho do programa MDFluxo na solução dos cacos clínicos em oftalmologia com os estudantes de medicina

\begin{tabular}{|c|c|c|c|c|}
\hline \multicolumn{5}{|c|}{ Teste estatístico de Friedman } \\
\hline $\begin{array}{c}\text { Hipótese } \\
\text { Diagnóstica }\end{array}$ & Etiologia & Conduta & $\begin{array}{l}\text { Tempo para finalizar } \\
\text { o caso clínico }\end{array}$ & $\begin{array}{c}\text { Somatória } \\
\text { dos resultados }\end{array}$ \\
\hline $\begin{array}{l}\chi^{2}=1,720 \\
(p=0,42)\end{array}$ & $\begin{array}{r}\chi^{2}=9,140 \\
(p=0,01)\end{array}$ & $\begin{array}{l}\chi^{2}=6,140 \\
(p=0,05)\end{array}$ & $\begin{array}{l}\chi^{2}=4,630 \\
(p=0,10)\end{array}$ & $\begin{array}{l}\chi^{2}=8,400 \\
(p=0,01)\end{array}$ \\
\hline $\begin{array}{l}\text { Não apresentou } \\
\text { diferença entre as } \\
\text { avaliações }\end{array}$ & $\begin{array}{c}\text { Computador de } \\
\text { mão \& livro } \neq \text { sem } \\
\text { qualquer material } \\
\text { de apoio }\end{array}$ & $\begin{array}{c}\text { Livro } \neq \text { Computador } \\
\text { de mão } \neq \text { sem } \\
\text { qualquer Material de } \\
\text { apoio }\end{array}$ & $\begin{array}{c}\text { Livro } \neq \text { Computador } \\
\text { de mão \& sem } \\
\text { qualquer material de } \\
\text { apoio }\end{array}$ & $\begin{array}{l}\text { Não apresentou } \\
\text { diferença entre as } \\
\text { avaliações }\end{array}$ \\
\hline
\end{tabular}

O teste estatístico de Friedman32 aplicado nestes dados não apresentou diferença significativa entre os dados. $O$ valor do qui-quadrado foi de $\chi^{2}=10,836$ e o valor de $\mathrm{p}=0,287$. Ou seja, as notas de satisfação dos estudantes com o uso do programa MDFluxo foram equivalentes entre si.

Os dados recuperados do arquivo de log na base de dados do programa MDFluxo foram tabulados e uti- lizados para observar se existe correlação entre a quantidade de itens navegados pelos estudantes durante a utilização do programa com a nota de acerto destes estudantes na solução do caso clínico. A Tabela 4 apresenta os dados recuperados do arquivo de log.

Para observar se existe correlação entre a quantidade de itens navegados com a porcentagem de acerto dos estudantes na avaliação de desempenho do pro- 


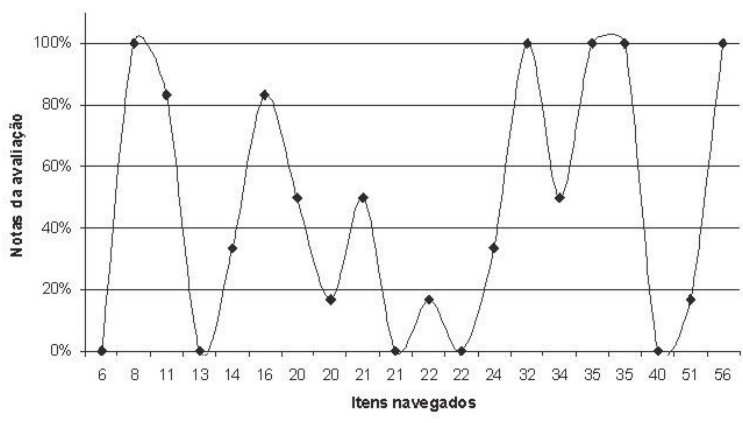

Figura 2: Gráfico com a porcentagem de acerto dos estudantes de medicina na avaliação de desempenho do programa MDFluxo e a quantidade de itens navegados

grama MDFluxo foi construído o gráfico onde os estudantes aparecem como um ponto identificando sua porcentagem de acerto na solução do caso clínico e sua quantidade de itens navegados no programa, como apresentado na figura 2.

Por meio deste gráfico é possível observar que os estudantes que navegaram 8 e 56 itens obtiveram acerto igual a $100,00 \%$ (nota 3,0$)$. Em outro exemplo, os estudantes que navegaram 6 e 40 itens obtiveram acerto igual a $0,00 \%$ (nota 0,0$)$. Ou seja, a porcentagem de acerto na solução do caso clínico não apresenta nenhuma correlação com a quantidade de itens navegados durante a utilização do programa, já que as mesmas porcentagens de acerto foram obtidas por estudantes que navegaram quantidades diferentes de itens.

Além do gráfico, foi aplicado o cálculo de correlação nestes dados. Caso exista uma correlação entre os dados o valor de $\mathrm{R} 2$ deve ser o mais próximo possível de 1,00 . O valor do cálculo de correlação dos dados foi de $\mathrm{R} 2=0,20$. Com isso pode-se afirmar que não existe correlação entre a quantidade de itens navegados e a porcentagem de acerto, ou seja, o resultado do estudante na solução do caso clínico não depende da quantidade de itens navegados.

A Figura 3 apresenta o gráfico com a quantidade de respostas sobre as notas de satisfação dos estudantes com o uso do programa MDFluxo na solução do caso clínico.

Pode-se observar neste gráfico que o programa MDFluxo obteve boa avaliação (nota 4) dos estudantes com 86 repostas, ótimo (nota 5) com 36 respostas e satisfatórias (nota 3) com 43 respostas. A quantidade de avaliação ruim (nota 2) e péssimo (nota 1) é inferior à quantidade de respostas satisfatória, bom e ótimo (35 para $165)$ respectivamente, ou seja, $17,5 \%$ dos estudantes rejeitaram o uso do programa MDFluxo e $82,5 \%$ aceitaram o uso do programa na solução dos casos clínicos.

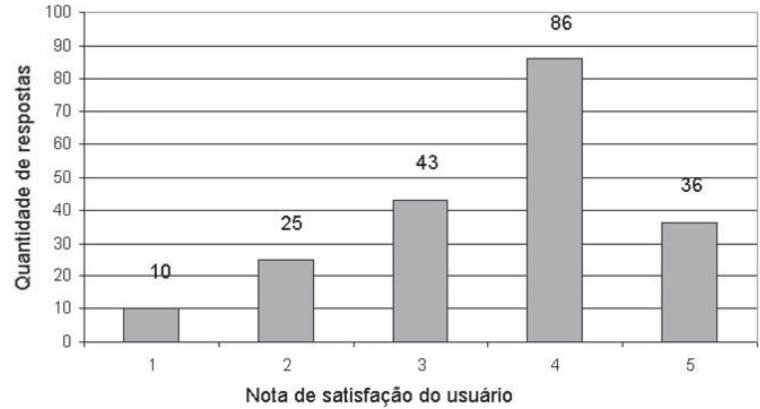

Figura 3: Gráfico com a quantidade de respostas das notas atribuídas pelos estudantes para o nível de satisfação com o uso do programa MDFluxo

Tabela 3

Média das notas atribuídas pelos estudantes de medicina para avaliar o desempenho do programa MDFluxo e suas respectivas porcentagens

Média das notas nas questões avaliadas

\begin{tabular}{lcc}
\hline Questões & $\begin{array}{c}\text { Notas de } \\
\text { satisfação }\end{array}$ & Porcentagem \\
\hline Q5 Eficácia & 3,25 & $65,00 \%$ \\
Q6 Eficiência & 3,60 & $72,00 \%$ \\
Q7 Confiabilidade & 3,70 & $74,00 \%$ \\
Q8 Consistência & 3,70 & $74,00 \%$ \\
Q9 Funcionalidade & 3,30 & $66,00 \%$ \\
Q10 Facilidade & 3,65 & $73,00 \%$ \\
Q11 Navegabilidade & 3,70 & $74,00 \%$ \\
Q12 Agilidade & 3,70 & $74,00 \%$ \\
Q13 Objetividade & 3,75 & $75,00 \%$ \\
Q14 Qualidade & 3,30 & $66,00 \%$ \\
\hline
\end{tabular}

\section{DısCUSSÃo}

O teste estatístico de Friedman ${ }^{(32)}$ aplicado nos dados obtidos na avaliação do programa MDFluxo os itens hipótese diagnóstica e somatória dos resultados não apresentaram diferenças significativas entre as avaliações realizadas com o uso do livro e sem qualquer material de apoio. Na etiologia, conduta e tempo para finalizar o caso clínico o uso do computador de mão com o programa MDFluxo e o uso do livro(27) se mostraram equivalentes entre si.

Durante as avaliações, estavam armazenados na base de dados do programa MDFluxo apenas 15 dos 55 fluxogramas do livro em formato XML, destes apenas 3 foram revisados por especialistas do departamento de 
Tabela 4
Dados recuperados do arquivo de log com os itens navegados pelos estudantes de medicina no computador de mão com o programa MDFluxo durante sua avaliação

\begin{tabular}{cccc}
\hline \multicolumn{2}{c}{ Dados recuperados do arquivo de log do programa } & MDFluxo \\
\hline $\begin{array}{c}\text { Quantidade de } \\
\text { itens navegados }\end{array}$ & $\begin{array}{c}\text { Quantidade } \\
\text { de avanços }\end{array}$ & $\begin{array}{c}\text { Quantidade } \\
\text { de retornos }\end{array}$ & $\begin{array}{c}\text { Quantidade } \\
\text { de fluxogramas } \\
\text { selecionados }\end{array}$ \\
\hline 6 & & & 1 \\
8 & 6 & 0 & 1 \\
11 & 8 & 0 & 1 \\
13 & 9 & 2 & 1 \\
14 & 10 & 3 & 3 \\
16 & 11 & 3 & 2 \\
20 & 13 & 4 & 3 \\
20 & 13 & 7 & 4 \\
21 & 13 & 7 & 2 \\
21 & 14 & 7 & 2 \\
22 & 14 & 7 & 2 \\
22 & 15 & 7 & 2 \\
24 & 15 & 8 & 3 \\
32 & 16 & 12 & 4 \\
34 & 20 & 14 & 1 \\
35 & 20 & 14 & 3 \\
35 & 21 & 14 & 5 \\
40 & 21 & 17 & 3 \\
51 & 23 & 21 & 25 \\
56 & 29 & & 2 \\
\hline
\end{tabular}

oftalmologia da UNIFESP. Ou seja, o programa não possuía a mesma quantidade de informações clínicas que o livro ${ }^{(27)}$. Com base nas notas de acerto dos estudantes nas avaliações é possível que a diferença na quantidade de informações encontradas no programa se comparado ao livro ${ }^{(27)}$ tenha influenciado no desempenho destes estudantes na avaliação.

Como apenas 3 dos 15 fluxogramas do livro ${ }^{(27)}$ armazenados na base de dados do programa MDFluxo estavam revisados e com base nos dados da Tabela 4 é provável que os estudantes tenham selecionado fluxogramas não revisados. Isto pode ser observado pela quantidade de avanços e retornos dos estudantes em um mesmo fluxograma durante a utilização do programa na solução do caso clínico. Para melhorar o desempenho dos estudantes na solução dos casos clínicos com o uso do computador de mão com o programa MDFluxo é preciso um número maior de fluxogramas revisados em sua base de dados e mais informações para complementar seu conteúdo.

Os estudantes de medicina não tiveram acesso ao programa MDFluxo antes das avaliações, sendo realizada apenas uma apresentação do mesmo e suas funcio- nalidades no dia da realização da avaliação. Ou seja, os estudantes não apresentavam nenhum conhecimento anterior sobre o programa, o que possivelmente melhoraria no desempenho dos estudantes na solução dos casos clínicos.

Por não apresentarem este conhecimento prévio sobre o uso do programa e a forma como as informações clínicas são apresentadas, tendo início com a escolha do segmento e finalizando com a ação médica selecionada, os estudantes podem ter achado o uso do programa complexo durante a navegação. Assim como no estudo realizado por Grad et al. ${ }^{(26)}$, a forma de apresentação das informações pode ter influenciado na compreensão do usuário.

Por outro lado, alguns estudantes conheciam o livro ${ }^{(27)}$ e a forma como a informação clínica é apresenta$\mathrm{da}$, por isso levaram menos tempo para finalizar o caso clínico se comparado ao uso do programa MDFluxo. Os estudantes que não utilizaram nenhum material de apoio (computador de mão com o programa MDFluxo ou livro) utilizou apenas o conhecimento prévio sobre o assunto abordado em cada caso clínico, com isso levaram menos tempo para finalizar o caso clínico. 
Assim como o programa MDFluxo, os programas FirstConsult, InfoRetriever e UpToDate são programas que fornecem informação de diagnóstico baseado em evidência de literaturas médicas digitalizadas em formato texto para computadores de mão. Os usuários que utilizaram estes programas apresentaram satisfação com o seu uso e aplicação ${ }^{(33)}$. Apesar de não apresentarem bons resultados, com o uso do programa MDFluxo na solução dos casos clínicos, a maioria dos estudantes de medicina aprovaram sua utilização.

Nos últimos anos o uso de materiais médicos de apoio, como livros, revistas, tutoriais, artigos e referências bibliográficas em formato digital, para computadores de mão têm aumentado gradativamente ${ }^{(19)}$.

Os autores concordam que o uso do computador de mão com o programa MDFluxo foi superior em relação ao uso do livro se comparado a não utilização de qualquer material de apoio. Apesar dos resultados, a avaliação com os estudantes de medicina não apresentou evidências de que o uso do computador de mão, comparado ao uso do livro, auxilie no aprendizado e retenção do conhecimento médico pelos estudantes ${ }^{(26,34)}$.

É possível que um estudo em longo prazo, com mais informações clínicas armazenadas na base de dados do programa MDFluxo e um treinamento no uso do programa, com os estudantes de medicina, melhore o nível de satisfação dos usuários com o uso do programa e os resultados na avaliação de desempenho do mesmo.

\section{Conclusão}

Foi construído um programa para computador de mão - aqui denominado MDFluxo - para auxiliar na solução de casos clínicos em oftalmologia para estudantes de medicina. Com base nos resultados apresentados neste artigo, computador de mão com o programa MDfluxo foi equivalente ao uso do livro ${ }^{(27)}$ como material de apoio aos estudantes de medicina.

Uma avaliação de satisfação realizada com os estudantes que utilizaram o computador de mão com o programa MDFluxo resultou em 82,5\% de aprovação pelos voluntários no experimento.

\section{Agradecimentos}

Agradecemos ao apoio institucional do Departamento de Oftalmologia e Setor de Tecnologia em Informação (STI), Departamento de Informática em Saúde (DIS), Universidade Federal de São Paulo (UNIFESP); também aos médicos e estudantes de medicina voluntários participantes da pesquisa. Agradecemos também a assessoria do Departamento de Medicina Preventiva e a colaboração do Prof. Dr. Neil Ferreira Novo e a Prof ${ }^{a}$ Dra. Yara Juliano na organização e análise dos dados coletados nesta pesquisa.

\section{Abstract}

Objective: In the last decade the handheld computers have been popularized among health professionals, on the USA, for instance, among $60-70 \%$ of medical students already use them. Make clinical information available on these devices can aid teaching medical practices, however, the way they are structured may influence students' satisfaction and learning. The purpose of this paper is to present MDFluxo evaluation, a program developed in handheld computers to aid ophthalmology teaching. Methods: The program was developed with structured flowcharts extracted from The Ophthalmology Guide book. The assessment was placed comparing 20 medical under-graduating students' performance solving clinical cases aided by the program, a book, or no material. Results: The results shown that the performance of students in the evaluation with book was equivalent to MDFluxo in etiology $(p<0.01)$ and conduct $(p<0.05)$ on clinical cases solving and better performance than without the use any material. Conclusion: On MDFluxo user satisfaction evaluation $82.5 \%$ of students approved it's use on clinical case solving.

Keywords: Computers, handheld; Medical students; Ophthalmology; Employee performance appraisal

\section{ReferêNCIAS}

1. Fischer S, Stewart TE, Mehta S, Wax R, Lapinsky SE. Handheld computing in medicine. J Am Med Inform Assoc. 2003;10(2):139-49.

2. Greiver M, Drummond N, White D, Weshler J, Moineddin R; North Toronto Primary Care Research Network (Nortren). Angina on the Palm: randomized controlled pilot trial of Palm PDA software for referrals for cardiac testing. Can Fam Physician. 2005;51:382-3.

3. Matthew AG, Currie KL, Irvine J, Ritvo P, Santa Mina D, Jamnicky $\mathrm{L}$, et al. Serial personal digital assistant data capture of healthrelated quality of life: a randomized controlled trial in a prostate cancer clinic. Health Qual Life Outcomes. 2007;5:38.

4. $\mathrm{Ng} \mathrm{CH}, \mathrm{Ng} \mathrm{CW}$, Soh E, Yeo JF. The PDA and the oral maxillofacial surgeon. Singapore Dent J. 2004;26(1):7-9.

5. Grasso MA, Yen MJ, Mintz ML. Survey of handheld computing among medical students. Comput Methods Programs Biomed. 2006;82(3):196-202.

6. King RV, Murphy-Cullen CL, Mayo HG, Marcee AK, Schneider GW. Use of computers and the Internet by residents in US family medicine programmes. Med Inform Internet Med. 2007;32(2):149-55. 
7. Hatfield AJ, Bangert MP. Implementation of the Clinical Encounters Tracking system at the Indiana University School of Medicine. Med Ref Serv Q. 2005;24(4), pp. 41-58.

8. Sutton J, Stockton L, McCord G, Gilchrist VJ, Fedyna D. Handheld computer use in a family medicine clerkship. Acad Med. 2004;79(11):1114-9.

9. Torre DM, Sebastian JL, Simpson DE. A PDA-based instructional tool to monitor students' cardiac auscultation during a medicine clerkship. Med Teach. 2005;27(6):559-61.

10. Joy S, Benrubi G. Personal digital assistant use in Florida obstetrics and gynecology residency programs. South Med J. 2004;97(5):430-3.

11. Luo J. Portable computing in psychiatry. Can J Psychiatry. 2004;49(1):24-30. Comment in: Can J Psychiatry. 2004:49(1):1-3.

12. Wilden J, Riley RH. Personal digital assistant (PDA) use amongst anaesthetists: an Australian survey. Anaesth Intensive Care. 2005;33(2):256-60.

13. Johnston WK 3rd, Patel BN, Low RK, Das S. Wireless teleradiology for renal colic and renal trauma. J Endourol. 2005;19(1):32-6.

14. Shirima K, Mukasa O, Schellenberg JA, Manzi F, John D, Mushi A, et al. The use of personal digital assistants for data entry at the point of collection in a large household survey in southern Tanzania. Emerg Themes Epidemiol. 2007;4:5.

15. Kho A, Henderson LE, Dressler DD, Kripalani S. Use of handheld computers in medical education. A systematic review. J Gen Intern Med. 2006;21(5):531-7. Review.

16. Honeybourne C, Sutton S, Ward L. Knowledge in the Palm of your hands: PDAs in the clinical setting. Health info Libr J. 2006;23(1):51-9.

17. Llorente Pendás JL, Alvarez Marcos CA, Muñiz Morán J, Martínez Nistal A, Sampedro Nuño A, Suárez Nieto C. [Otorhinolaryngological consultation material in PDA: SEORL-PDA]. Acta Otorrinolaringol Esp. 2006;57(4):15560. Spanish.

18. Stroud SD, Erkel EA, Smith CA. The use of personal digital assistants by nurse practitioner students and faculty. J Am Acad Nurse Pract. 2005;17(2):67-75.

19. Nishino M, Busch JM, Wei J, Barbaras L, Yam CS, Hatabu H. Use of personal digital assistants in diagnostic radiology resident education. Acad Radiol. 2004;11(10):1153-8.

20. Rudkin SE, Langdorf MI, Macias D, Oman JA, Kazzi AA. Personal digital assistants change management more often than paper texts and foster patient confidence. Eur J Emerg Med. 2006;13(2):92-6.

21. Ibanez Collado C, Juarez Giménez JC, Monterde Junyent J. Bases de datos clínicas y farmacológicas para asistentes personales digitales. An Pediatr (Barc). 2005;62(6):564-72.
22. Rothschild JM, Lee TH, Bae T, Bates DW. Clinician use of a palmtop drug reference guide. J Am Med Inform Assoc. 2002;9(3):223-9.

23. Nakata N, Kandatsu S, Suzuki N, Fukuda K. Informatics in Radiology (infoRAD): mobile wireless DICOM server system and PDA with high-resolution display: feasibility of group work for radiologists. Radiographics. 2005;25(1):273-83.

24. Marozas V, Jurkonis R, Kazla A, Lukoševicius M, Lukoševicius A, Gelzinis A, Jegelevicius D. Development of teleconsultations systems for e-health. Stud Health Technol Inform. 2004;105:337-48.

25. Scheinfeld NS. Creating and utilizing a multimedia dermatology medical record for a PocketPC personal digital assistant. Skinmed. 2005;4(1):33-7.

26. Grad RM, Meng Y, Bartlett G, Dawes M, Pluye P, Boillat M, et al. Effect of a PDA-assisted evidence-based medicine course on knowledge of common clinical problems. Fam Med. 2005;37(10):734-40. Comment in: Fam Med. 2005;37(10):741-2.

27. Schor P, Chamon W, Belfort Júnior R. Guia de oftalmologia. São Paulo: Manole; 2004.

28. Kara José AC, Passos LB, Kara José FC, Kara José N. Ensino extracurricular em Oftalmologia: grupos de estudos / ligas de alunos de graduação. Rev Bras Educ Méd. 2007;31(2):166-72.

29. Daum B, Merten U. Arquitetura de sistemas com XML. Rio de Janeiro: Campus; 2002.

30. Shiffman RN, Michel G. Toward improved guideline quality: using the COGS statement with GEM. Stud Health Technol Inform.2004;107(Pt1):159-63.

31. Extreme Programming (XP). The rules and practices of extreme programming [Internet]. [cited 2007 Ago 27]. Available from: http://www.extremeprogramming.org/rules.html .

32. Siegel S, Castellan Jr NJ. Nonparametric statistics for the behavioral sciences. 2a ed. New York: McGraw-Hill; 1988. p. 399.

33. Burkiewicz JS, Vesta KS, Hume AL. Update in handheld electronic resources for evidence-based practice in the community setting. Ann Pharmacother. 2005;39(12):2100-4.

34. Lawler FH, Cacy J. Utilization and value of personal digital assistants on an epidemiology final examination. Teach Learn Med. 2005;17(2):166-8.
Endereço para correspondência
Rua Botucatu, $\mathrm{n}^{\circ} 862$
CEP 04023-062 - São Paulo (SP), Brasil
Tel: (11) 5574-0158 - Fax (11) 5574-0110
vladimir.camelo@uol.com.br 